\title{
Análisis psicométrico del Inventario Multidimensional de Infidelidad, en estudiantes de educación superior en Cartagena, Colombia.
}

\section{Psychometric analysis of the Multidimensional Inventory of Infidelity, in higher education students in Cartagena, Colombia.}

\author{
Elías Rafael Geney Castro ${ }^{1}$, Mariana Inés Tezón ${ }^{2}$, David Javier Fortich Pérez ${ }^{1}$, Silvia Baeza ${ }^{3}$ \\ 1Universidad Tecnológica de Bolívar, Colombia \\ ${ }^{2}$ Fundación Universitaria Tecnológico Comfenalco, Colombia \\ 3Universidad del Salvador, Argentina \\ (Rec.: febrero de 2018 - Acep.: mayo de 2018)
}

\begin{abstract}
Resumen
La infidelidad es un incumplimiento de un convenio donde se infringen los acuerdos de exclusividad relacional. Es uno de los motivos más frecuentes del deterioro en las relaciones y en muchos casos quebranta la calidad de vida, la integridad y la salud. Frente a sus implicaciones es necesario contar con instrumentos capaces de evaluar las diferentes manifestaciones y consecuencias. El objetivo de la investigación fue realizar una exploración de las propiedades psicométricas del Inventario Multidimensional de Infidelidad de Romero, Rivera y Díaz. Con un diseño descriptivo instrumental. La muestra de 500 individuos, con media 20.3 años y $73.2 \%$ de sexo femenino, se realizó análisis factorial exploratorio con rotación varimax. Se determinaron factores con autovalores mayores a 1 y que la varianza total explicara más del $50 \%$. Los índices de consistencia interna con la fórmula Alfa de Cronbach se ubicaron entre 0.818 y 0.978. Los resultados indican que el instrumento posee la capacidad de medir diferentes categorías de la infidelidad, a través de 4 subescalas; Conducta Infiel, Motivos de Infidelidad, Conceptos de Infidelidad y Consecuencias de infidelidad. El inventario presentado tiene propiedades satisfactorias de validez y confiabilidad que lo hacen útil en la evaluación de la infidelidad en el contexto.
\end{abstract}

Palabras Claves: Infidelidad, Confiabilidad, análisis factorial, Psicometría, Colombia.

\begin{abstract}
Infidelity is a breach in an agreement where the exclusivity arrangements are violated. It is one of the most frequent reasons for deterioration in relationships and in many cases violates the quality of life, integrity and health. Facing its implications, it is necessary to have instruments capable of evaluating the different manifestations and consequences. The objective of this research was to carry out an exploration of the psychometric properties of the Multidimensional Infidelity Inventory of Romero, Rivera and Diaz, with a descriptive instrumental design. We carried out an exploratory factor analysis with varimax rotation in a sample of 500 individuals, with a mean of 20.3 years and a $73.2 \%$ of females. We determined factors with eigenvalues greater than 1 and the total variance explained more than $50 \%$. The internal consistency indices with Cronbach's Alpha formula were between 0.818 and 0.978 . The results indicated that the instrument is able to measure different categories of infidelity through 4 subscales: unfaithful behavior, reasons for infidelity, infidelity concepts and infidelity consequences. The presented inventory has satisfactory validity and reliability properties that make it useful in the evaluation of infidelity in context.
\end{abstract}

Key Words: Infidelity, Reliability, Factor Analysis, Psychometrics, Colombia

correspondencia: Elias Rafael Geney C. Universidad Tecnológica de Bolívar, Colombia. email: egeney@utb.edu.co 


\section{Introducción}

La infidelidad es un fenómeno que puede estar presente en el acontecer de la vida conyugal. Es definida por Pittman (2003) como una defraudación, la violación de un convenio o la traición de una relación, donde el componente más significativo es la violación de la exclusividad. Para Camacho (2004), la infidelidad se asocia a la transgresión de un acuerdo conyugal, que está determinado por la historia misma de cada individuo. Scheinkman y Fishbane (2004) indican que la infidelidad representa una traición de la relación primaria, cuyos conflictos se pueden originar en el cumplimiento de las expectativas, el compromiso y verdad. En una línea similar, Weiser, Lalasz, Weigel y Evans (2014) plantean que la conducta infiel se asocia con un tipo de actitud deshonesta.

La infidelidad como proceso problemático en la vida conyugal, se puede asociar con deterioro físico y emocional, ocasionando un malestar psicológico general, e incluso, problemas de salud mental, como, por ejemplo, síntomas depresivos, recuerdos dolorosos e intrusivos, cólera, amenazas al sentido de seguridad, trastorno bipolar, alcoholismo y trastornos de la alimentación (García, 2002; Gottman, 1998; Christensen \& Heavey,1999; Finchman \& Beach, 1999; Leary \& springer, 2001; Gordon Snyder \& Baucom, 2005).

De igual forma, Whisman (1999) señala que en las relaciones donde hay elevados índices de insatisfacción se encuentran niveles elevados de depresión, estrés y distimia. Dichos conflictos también se asocian con algunas enfermedades cardíacas, enfermedades crónicas y afectaciones en el sistema inmune.

Gordon, Baucon y Snider (2004) también manifiestan que el proceso de infidelidad puede ocasionar dificultades como depresión y síntomas de estrés post traumático, además de cólera, vergüenza y alteraciones constantes en la estabilidad marital, convirtiéndose en uno de los factores que, desde lo psicológico, lo relacional y lo físico, genera múltiples consecuencias negativas a nivel relacional que conlleva al deterioro o ruptura definitiva de la relación de pareja, por lo tanto puede predecir divorcios. De igual manera, se constituye en un factor de riesgo para contraer enfermedades de transmisión sexual (Janus \& Janus, 1993; Rogers, 1997; Collazos, Echeverry, Molina, Canaval \& Valencia, 2005; Nall, Beadle, Newell \& Helm, 2008; Balderrama-Durbin, Stanton, Snyder, Cigrang, Talcott, Smith Slep \& Cassidy, 2017).

Por otra parte, Camacho (2004) describe que esta problemática se percibe como la ruptura de un contrato, acuerdo implícito o explícito, en el cual uno de los dos miembros en una pareja, tiene algún tipo de relación con una tercera persona. Zumaya, Brown y Baker (2003), afirman que las conductas infieles no son exclusivamente sexuales, ni tienen que ver con el amor al cónyuge; no obstante, cuando uno de los miembros es infiel, en la pareja se rompe la confianza y se produce inseguridad, ya que en la estructura relacional se establecen sentimientos de abandono.

La infidelidad según Zola (2004), incluye los actos de traición como el sexo virtual, las relaciones emocionales íntimas, las relaciones secretas, el uso sigiloso de pornografía, la traición financiera, además de una relación sexual física con un tercero. Estas traiciones pueden ser consideradas como viola- ciones de expectativas de la exclusividad emocional y física, $y$ al descubrirlas se implican sentimientos de engaño que probablemente causarán desilusión y problemas de relación adicionales (Whisman \& Wagers, 2005; Guitar, Geher, Kruger, Garcia, Fisher \& Fitzgerald, 2017).

Estudiar la infidelidad es muy importante debido a la connotación que tiene para los individuos, las relaciones y la vida de pareja. Este fenómeno constituye uno de los motivos de celos más frecuentes, contribuyendo de esta manera en el deterioro de las relaciones, ya que afecta distintas esferas en el individuo y en muchos casos, quebranta los esquemas de valoración positiva de sí mismo y del otro, la calidad de vida, la integridad y la salud (Sagarin, Becker, Guadagno, Nicastle \& Millevoi, 2003; Feeney, 2005; Espinoza, Correa \& Garcia, 2014). A pesar que existen una variedad de estudios acerca de la infidelidad, es muy poco explorado en población universitaria. Canal y Carrillo (2013), manifiestan que los estudiantes universitarios dan un significado importante a sus relaciones de pareja, ya que estas contribuyen en la construcción de sus proyectos de vida a nivel personal, conyugal, familiar y laboral, donde se propicia una sana aceptación de sí mismo y de los contextos que los rodean. Frente a tantas implicaciones, es necesario contar con instrumentos capaces de evaluar las diferentes manifestaciones y consecuencias del fenómeno.

En ese orden de ideas, Barbero, Vila y Holgado (2008) señalan que para evaluar bien y coherentemente un fenómeno, se necesita llevar a cabo un proceso de validación, que permita adaptar cada patrón de medición a las características propias del contexto cultural y así poder elaborar nuevos baremos. Por tal razón, todo instrumento de medida debe ser adecuado para el fenómeno que se quiera medir, y como requisitos esenciales deberá: ser teóricamente justificable e intuitivamente razonable; ser capaz de medir aquellas características que pretenden medir y no otras; ser confiable y preciso, con un mínimo de error en la medida; ser sensible, siendo capaz de medir cambios tanto en los diferentes individuos como en la respuesta de un mismo individuo a través del tiempo; y por último, deberá delimitar claramente sus componentes de manera que cada uno contribuya al total de la escala de forma independiente (Arribas, 2004).

Con respecto a la temática de infidelidad, existen instrumentos que en su mayoría se orientan a medir conducta infiel, actitudes hacia la infidelidad y justificación. Entre los instrumentos más relevantes está la Escala de Actitudes Hacia la Exclusividad Marital, que consta de 7 reactivos tipo Likert con 5 opciones de respuesta, y evalúa las percepciones hacia la aceptación de relaciones extramaritales tanto sexuales como no sexuales con miembros del sexo opuesto. La escala obtuvo un Alpha total de 0.88 (Weis \& Felton, 1987).

Otro cuestionario utilizado es el de Razones para el Involucramiento Extramarital, el cual consta de 17 reactivos tipo Likert, con 4 opciones de respuesta. Este cuestionario obtuvo su validez mediante un análisis factorial de componentes principales con rotación ortogonal, que arrojó 4 factores que explicaron el $74 \%$ de la varianza (Glass \& Wright, 1992).

Por otro lado, Banfield y McCabe (2001) diseñaron un instrumento que evalúa las intenciones para cometer una infidelidad sexual o emocional, en una escala tipo Likert de 7 puntos de respuesta con un Alpha total de 0.89 . 
Un cuarto instrumento es el Cuestionario de Infidelidad, instrumento que consiste en 24 reactivos tipo Likert con 5 opciones de respuesta, y que mide las posibles razones por las que un hombre o una mujer serían infieles. Consta de seis factores: Legitimidad, Seducción, Normalización, Sexualidad, Antecedentes Sociales, y Búsqueda de Sensaciones, que explican el $45.11 \%$ de la varianza (Yeniceri \& Kökdemir, 2006).

Otra evaluación es la Escala de Actitud de la Población Mexicana ante la Infidelidad y la Persona Infiel. Tiene 30 reactivos, divididos en tres factores que se refieren a la actitud hacia la persona infiel explicando el $54 \%$ de la varianza, y 2 factores que se refieren a la actitud hacia la infidelidad, que explican el $60 \%$ de la varianza (Bonilla, 1993).

Finalmente, el Inventario Multidimensional de Infidelidad (IMIN) diseñado por Romero, Rivera y Diaz-Loving, está constituido por 4 subescalas: Conducta Infiel, Concepto de Infidelidad, Motivos de Infidelidad y Consecuencias de Infidelidad, y un total de 175 ítems. Este instrumento fue seleccionado porque amplía la medición de la infidelidad, ya que no solo mide la conducta infiel en todas sus manifestaciones, sino que también se enfoca en medir la connotación que la población en general tiene de la infidelidad, contemplando la evaluación de las consecuencias percibidas por la gente en torno a los motivos, el concepto y las consecuencias positivas o negativas atribuidas al fenómeno. Esto permite agrupar en un solo inventario la evaluación de la conducta infiel, el concepto que se tiene de la misma, las razones que se consideran importantes para la presencia del fenómeno y las implicaciones que conlleva (Romero, Rivera \& Díaz Loving, 2007).

Con base en lo anterior, el objetivo de investigación fue realizar una exploración de las propiedades psicométricas del Inventario Multidimensional de Infidelidad de Romero, Rivera y Díaz, en estudiantes de educación superior en Cartagena, Colombia.

\section{Método}

Con respecto a las características del estudio, se realizó un diseño descriptivo instrumental, para conocer las propiedades psicométricas del instrumento en una población determinada (Montero y León, 2007).

\section{Participantes}

El muestreo fue no probabilístico accidental, y la prueba fue aplicada a 500 personas en instituciones de educación superior en Cartagena de Indias (Colombia), todos adultos, con una media de 20.3 años de edad, IC de 95\% (20.06-20.55), $73.2 \%$ de sexo femenino y $25.6 \%$ masculino. La muestra cumple con los supuestos de Comrey y Lee (2013), quienes en la orientación para determinar la adecuación del tamaño muestral, consideran que son muy buenas las muestras que cuentan con 500 individuos o más.

\section{Instrumento}

El instrumento aplicado fue el Inventario Multidimensional de Infidelidad (IMIN) diseñado por Romero et al (2007). El inventario está constituido por 4 subescalas: Conducta Infiel, Concepto de Infidelidad, Motivos de Infidelidad y Consecuencias de Infidelidad. Con un total de 175 ítems, el formato de respuesta se contesta con una escala tipo Likert con 5 opciones.

\section{Procedimiento}

Inicialmente se revisó el lenguaje en el instrumento, con una revisión disciplinar por medio de un psicólogo y una revisión metodológica por parte de un comunicador social, ambos profesionales de la misma región donde se aplicó la prueba.

Posteriormente se prosiguió a realizar la aplicación con el consentimiento informado firmado por los participantes. Se continuó con la tabulación y se procedió con el proceso de validez de constructo y la confiabilidad por consistencia interna.

El proceso investigativo, de acuerdo a lo expresado por Carvajal y Vásquez (1993), consideró los aspectos éticos contemplados en la declaración de Helsinki en lo referente a la protección de la vida, dignidad, integridad, derecho a la autodeterminación, intimidad y confidencialidad de la información de las personas que participaron en la investigación. A su vez, la investigación cumplió con lo establecido en la ley 1.090 (2006), en el Título II, Numeral 9: "Investigación con participantes humanos: la decisión de acometer una investigación descansa en el juicio que hace cada psicólogo sobre cómo contribuir mejor al desarrollo de la Psicología y al bienestar humano. Por lo tanto, el psicólogo aborda la investigación respetando la dignidad y el bienestar de las personas que participan y con pleno conocimiento de las normas legales y de los estándares profesionales que regulan la conducta de la investigación con participantes humanos".

\section{Análisis de datos}

Para realizar un análisis factorial exploratorio (en adelante AFE), se verificaron los supuestos estadísticos necesarios, como la linealidad a través del determinante, cuyo valor debería estar cercano a cero, la correlación de las variables con la medida de adecuación muestral Kaiser Mayer Olkin "KMO", el cual se considera regular o aceptable cuando el valor es igual o superior a 0.700 o sobresaliente cuando es igual o superior a 0.800 , y la prueba de esfericidad de Barlett, cuyos valores obtenidos deben resultar significativos a un nivel $p<0.05$. De igual manera se evaluó que en la matriz de correlación anti-imagen los valores de los ítems en la diagonal fuesen superiores a 0.500, sino era un criterio para eliminarlo (Hair, Black, Babin, Anderson \& Tatham, 2006; Méndez \& Rondón, 2012; Frías-Navarro \& Soler, 2012; Pérez \& Medrano, 2010).

El AFE se realizó por componentes principales con rotación varimax para alcanzar la mayor implicación posible y facilitar la interpretación de la matriz. Se verificó la comunalidad de los ítems, que es la proporción de varianza de cada ítem explicada por los factores extraídos, corroborando que no fuera inferior a 0.400; si era inferior a ese valor, el criterio sería eliminarlo. Para la determinación de los factores, se tuvo presente autovalores mayores a uno, que la varianza total explicara más del $50 \%$, considerando además el gráfico de sedimentación donde la línea se hiciera horizontal, y que cada factor tuviera más de tres ítems con valores mayores a 0.300 en la matriz de estructura (Hair, Black, Babin, Anderson \& Tatham, 1998; Sepúlveda, 2010; Merenda, 1997). 
La consistencia interna se realizó con la fórmula del Alfa de Cronbach a la escala completa y a cada factor que resultaba del AFE, y se verificaba cada vez que se realizaba la eliminación de un ítem en el AFE para también justificar la eliminación por Alfa (Oviedo \& Arias, 2005).

Por último, se realizó la estandarización de las puntuaciones determinado tipificación de las puntuaciones de cada sub escala y por cada factor resultante.

\section{Resultados}

En el proceso de la revisión y análisis por los profesionales disciplinares y metodológicos no hubo necesidad de realizar modificaciones para su entendimiento. Luego se realizó una prueba piloto con 50 personas, cuyos resultados indicaron no realizar cambios en la redacción ni estructura.

\section{Subescala Conducta Infiel}

Esta sub-escala original tiene 48 ítems, con un índice de consistencia interna de 0.702 divididos en 4 factores (Infidelidad Sexual, Deseo de Infidelidad Emocional, Deseo de infidelidad Sexual, Infidelidad Emocional), los cuales se describen a continuación:

1. Infidelidad sexual: este factor da a conocer las conductas que denotan el mantenimiento de un vínculo sexual con otra persona además de la pareja primaria. Originalmente consta de 21 ítems con un alfa de Cronbach de 0.432 .

2. Deseo de Infidelidad Emocional: el segundo factor denota el deseo de un vínculo romántico con otra persona además de la pareja primaria, sin necesariamente llevarlas a cabo. Este factor inicialmente tiene 14 ítems un índice de consistencia interna con un alfa de Cronbach de 0.463 . 3. Deseo de Infidelidad Sexual: el tercer factor se denomina Deseo de Infidelidad Sexual y se define como el deseo de un vínculo sexual con otra persona además de la pareja primaria, sin necesariamente llevarlas a cabo. Este factor inicialmente tiene 8 ítems y una consistencia interna de 0.449 .

4. Infidelidad Emocional: el último factor de la subescala Conducta Infiel es la Infidelidad Emocional, y es definido como la conducta que denota el mantenimiento de un vínculo emocional romántico con otra persona además de la pareja primaria. Este factor inicialmente tiene 5 ítems con un índice de consistencia interna de 0.390.

Al realizar el AFE de esta subescala, el determinante fue 7,708E-12, el KMO 0.931, y la Prueba de esfericidad de Barlett puntuó estadísticamente significativa al nivel 0.0001. En la correlación anti-imagen, el ítem 21 estuvo por debajo de 0.500 por lo que se eliminó, mejorando el resultado en el alfa 0.713 , así como el KMO. En la varianza explicada, se encontraron doce factores que explican el $61.090 \%$. Se procedió a eliminar ítems por comunidades inferiores a 0.300 y cargas factoriales similares o negativas como los ítems $(8,9,12,16,19,22,30$, $34,38,40)$. En los siguientes AFE se eliminaron 15 ítems más por presentar comunalidades muy bajas, cargas factoriales similares y siempre verificando que mejoraran la consistencia interna de la prueba.

El último AFE con 23 Ítems, arrojó los siguientes resultados: el determinante fue de 2.513E-9; el KMO, 0.949; la prueba de esfericidad de Barlett estuvo estadísticamente significativa al nivel 0.0001; en la correlación anti-imagen los ítems marcaron por encima de 0.500 , y las comunalidades fueron superiores a 0.400 . Se encontraron 4 factores que explican el $71.654 \%$ de la varianza total explicada, cada factor quedó con varianzas explicadas entre $24.679 \%$ y $11,988 \%$, las cargas factoriales fueron superiores a 0.491 con un Alpha de Cronbach de 0.959 .

En el análisis se observaron resultados satisfactorios, por lo cual se decide dejar esta subescala con 23 ítems. La composición final se muestra en la Tabla 1.

Tabla 1. Composición final de la subescala Conducta Infiel

\begin{tabular}{|c|c|c|c|}
\hline FACTOR & ÍTEMS & ALFA & IC $95 \%$ \\
\hline \multirow[t]{9}{*}{ Infidelidad Sexual } & He tenido relaciones sexuales con otra(s) persona(s) además de mi pareja & 0.933 & $\begin{array}{c}(0.921- \\
0.943)\end{array}$ \\
\hline & He tenido sexo con otra(s) persona(s) además de mi pareja & & \\
\hline & $\begin{array}{l}\text { Me he involucrado sentimental y sexualmente con otra(s) persona(s) además de mi } \\
\text { pareja }\end{array}$ & & \\
\hline & He tenido momentos pasionales con otra(s) persona(s) además de mi pareja & & \\
\hline & He engañado a mi pareja con otra(s) persona(s) & & \\
\hline & Le he sido infiel a mi pareja & & \\
\hline & He tenido varias parejas a la vez & & \\
\hline & Me he involucrado emocionalmente y sexualmente en otra relación romántica & & \\
\hline & He buscado nuevas experiencias con otra(s) persona(s) además de mi pareja & & \\
\hline \multirow[t]{4}{*}{$\begin{array}{l}\text { Deseo de } \\
\text { Infidelidad Sexual }\end{array}$} & He deseado tener relaciones sexuales con otra(s) persona(s) además de mi pareja & 0.939 & $\begin{array}{c}(0.928- \\
0.949)\end{array}$ \\
\hline & He deseado tener contacto sexual con otra(s) persona(s) además de mi pareja & & \\
\hline & He deseado sexualmente a otra(s) persona(s) además de mi pareja & & \\
\hline & He tenido fantasías sexuales con otra(s) persona(s) además de mi pareja & & \\
\hline
\end{tabular}


He deseado a otra(s) persona(s) además de mi pareja

He abrazado apasionadamente a otra(s) persona(s) además de mi pareja

He deseado relacionarme afectivamente con otra(s) persona(s) además de mi pareja

Infidelidad

Emocional

Me he relacionado sentimentalmente con otra(s) persona(s)

0.818

$(0.787-$

He tenido otras pareja amorosas

Me he relacionado afectivamente con otra(s) persona(s) además de mi pareja

He amado a otra(s) persona(s) además de mi pareja

Deseo de Infidelidad

Emocional

Me he interesado en otra(s) persona(s) además de mi pareja

0.845

$(0.818$

$0.868)$

He coqueteado con otra(s) persona(s) además de mi pareja

He acariciado a otra(s) persona(s) además de mi pareja

\section{Subescala Motivos de Infidelidad}

La escala inicial está compuesta por 70 ítems, con un índice de consistencia interna de 0.978. Contiene 7 factores que explican el $62.8 \%$ de la varianza, los cuales se describen a continuación:

Insatisfacción en la relación primaria: atribuye la infidelidad a problemas de comunicación, atención e interés existentes dentro de la relación primaria (ítems del \#1 - 18, Alfa de Crombach 0.966).

1. Sexualidad: este factor imputa la infidelidad a la búsqueda de satisfacción de necesidades principalmente de índole sexual y lúdico (ítems del \# 19-35, Alfa de Crombach 0.957).

2. Inestabilidad Emocional y Social: la infidelidad es adjudicada a problemas de tipo personal principalmente de índole emocional (ítems del \#36 - 42, Alfa de Crombach 0.908).

3. Ideología y Normas: refiere la infidelidad a la estructura de valores e ideología del individuo, derivadas de su educación y medio (ítems del \#43 - 50, Alfa de Crombach 0.901).

4. Impulsividad: señala la infidelidad como una consecuencia de los errores o impulsos del individuo carente de control sobre sí mismo (ítems del \#51 -57, Alfa de Crombach 0.895).

5. Apatía: la infidelidad encubre el desamor y la apatía dentro de la relación primaria (ítems del \#58-66, Alfa de
Crombach 0.918).

6. Agresión: atribuye la infidelidad a sentimientos de coraje, enojo y venganza hacia la pareja primaria (ítems del \#67 - 70, Alfa de Crombach 0.849).

Al realizar el AFE de esta subescala, el determinante fue 1.64E029, el KMO, 0.959, la Prueba de esfericidad de Barlett puntuó estadísticamente significativa al nivel 0.0001 , en la correlación anti-imagen todos los Ítems estuvieron por encima de 0.500 , en las comunalidades todos los ítems superiores a 0.400 , y en la varianza explicada se encontraron diez factores que explican el $70.209 \%$. El Alpha de Cronbach resultó 0.978 y se eliminaron por cargas factoriales similares los ítems (14, 26, 28, $31,40,41,58,66)$. En los siguientes AFE se eliminaron 12 ítems más por la misma razón.

El último AFE, con 50 ítems, arrojó los siguientes resultados: el determinante fue de 91.000E-013; el KMO, 0.951; la prueba de esfericidad de Barlett estuvo estadísticamente significativa al nivel 0.0001; en la correlación anti-imagen los ítems marcaron por encima de 0.500 , y las comunalidades fueron superiores a 0.400 . Se encontraron 7 factores que explican el $69.611 \%$ de la varianza total explicada, y cada factor quedó con varianzas explicadas entre $19.429 \%$ y 5,379\%, las cargas factoriales fueron superiores a 0.475 , con un Alpha de Cronbach de 0.970 .

En este análisis se observaron resultados satisfactorios, por lo cual se decide dejar esta subescala con 50 ítems. La composición final se muestra en la Tabla 2.

Tabla 2. Composición final de la subescala Motivos de Infidelidad

\begin{tabular}{|c|c|c|c|}
\hline Factores & Ítems & Alpha & IC $95 \%$ \\
\hline \multirow{10}{*}{$\begin{array}{l}\text { Insatisfacción con la Relación } \\
\text { Primaria }\end{array}$} & Falta de cariño en mi relación de pareja & 0.958 & $(0.952-0.963)$ \\
\hline & Falta de amor en mi relación de pareja & & \\
\hline & Falta de interés en mi relación de pareja & & \\
\hline & Falta de comunicación con mi pareja & & \\
\hline & Falta de atención por parte de mi pareja & & \\
\hline & Descuido en mi relación de pareja & & \\
\hline & Falta de comprensión por parte de mi pareja & & \\
\hline & Insatisfacción con mi relación de pareja & & \\
\hline & Falta de compromiso en mi relación de pareja & & \\
\hline & Falta de reconocimiento por falta de mi pareja & & \\
\hline
\end{tabular}




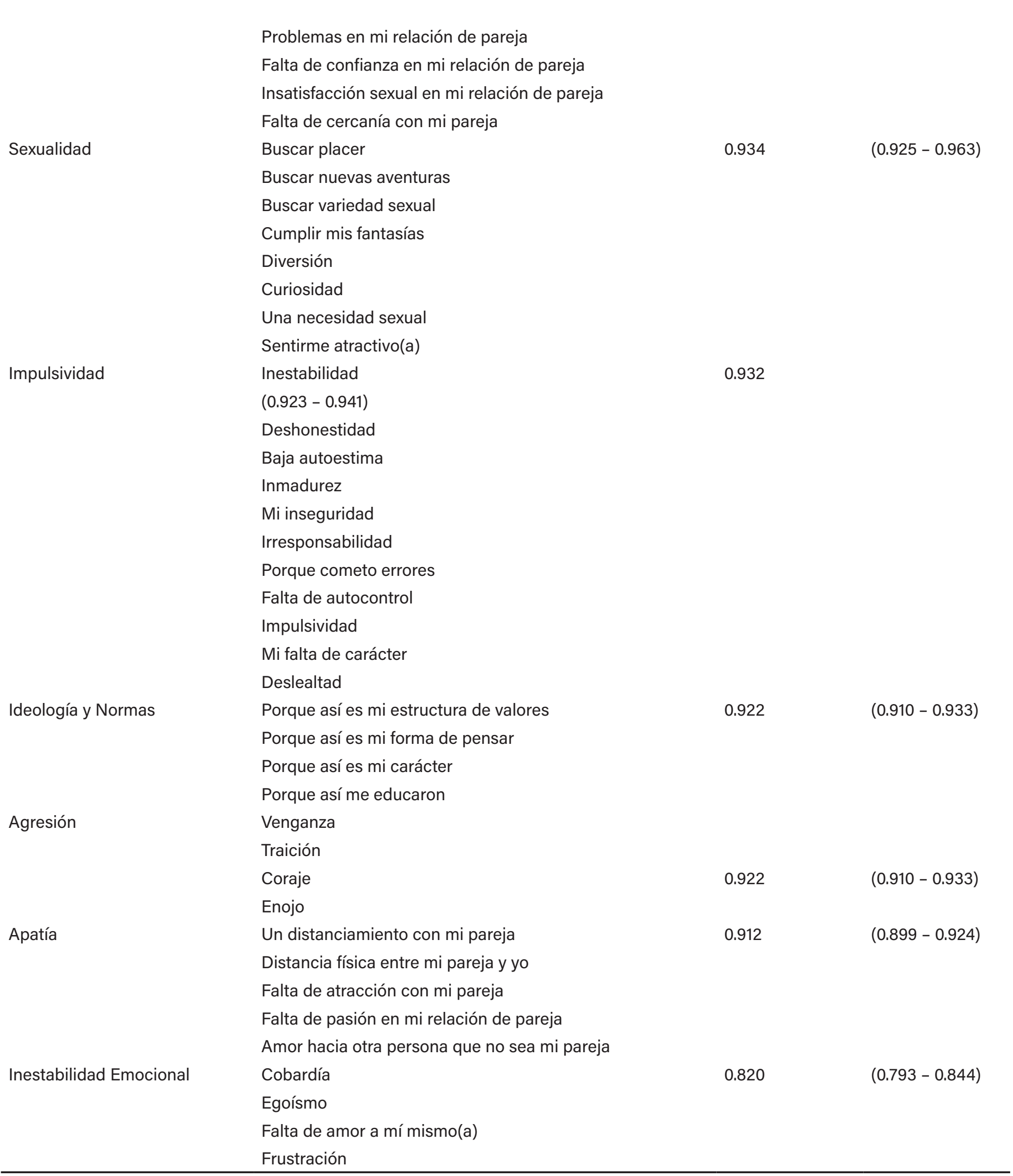

\section{Subescala Concepto de Infidelidad}

Inicialmente dicha escala está compuesta por 44 ítems, contando con un índice de consistencia interna de 0.967. Asimismo, estaba estructurada con 6 factores que explican el $69.1 \%$ de la varianza, los cuales se describen a continuación:

1. Trasgresión a la Relación: conceptualiza la infidelidad como una falta al vínculo exclusivo establecido dentro de la relación primaria, cuyo índice de consistencia interna era de 0.955 .

2. Sentimiento de Pérdida: la infidelidad es percibida como un acto derivado de un sentimiento negativo individual, con una consistencia interna de 0.911 .

3. Insatisfacción: significa la infidelidad como una falta de seguridad y congruencia por parte de los individuos que 
la llevan a cabo, contando con una consistencia interna inicial de 0.938 .

4. Pasión: este factor etiqueta la infidelidad como una aventura llena de pasión, deseo y novedad, manifestando una consistencia interna de 0.892 .

5. Inseguridad: la infidelidad se concibe como un hecho derivado de inseguridad, confusión, cobardía y egoísmo por parte de los individuos que la llevan a cabo, e inicialmente tenía una consistencia interna de 0.879 .

6. Amor hacia otro: la infidelidad es conceptualizada como el encuentro con el amor, el cariño y la comprensión perdidos dentro del vínculo primario, mostrando una consistencia interna de 0.901 .

Al realizar el AFE, se eliminaron los ítems 9, 19, 23, 24, 26, 27,
$29,37,39$ y 44 por cargas factoriales similares. Con los 34 ítems restantes, el Alpha de Cronbach fue 0.950, el Determinante 1.69E-011, el KMO, 0.939, y la Prueba de esfericidad de Barlett fue Estadísticamente significativa al nivel 0.0001. En la matriz de correlación anti-imagen todos los ítems estuvieron por encima de 0.500 , y en la varianza total explicada se encontraron seis factores, proponiéndose modificar el factor Inseguridad por uno denominado Problemas de Comunicación. El primer factor explica el 18,791\%; el segundo, el 12,923\%; el tercero, $12,496 \%$; el cuarto, $10.463 \%$; el quinto, $6,980 \%$; y el sexto, $5,943 \%$, para un total de $67.596 \%$.

En este análisis se observaron resultados satisfactorios, por lo cual se decide dejar esta subescala con 34 ítems. La composición final de esta subescala se muestra en la tabla 3.

Tabla 3. Composición final de la subescala Concepto de Infidelidad

\begin{tabular}{|c|c|c|c|c|}
\hline Factores & Ítems & Comunalidades & Alpha & IC 95\% \\
\hline \multirow{9}{*}{$\begin{array}{l}\text { Transgresión a la } \\
\text { relación }\end{array}$} & Falsedad & 652 & 0.938 & $(0.930-0.946)$ \\
\hline & Deshonestidad & ,805 & & \\
\hline & Falta de respeto & ,710 & & \\
\hline & Falta de compromiso & 699 & & \\
\hline & Desconfianza &, 534 & & \\
\hline & Desamor & 682 & & \\
\hline & Hipocresía & 689 & & \\
\hline & Engaño & ,772 & & \\
\hline & Traición & ,775 & & \\
\hline \multirow[t]{5}{*}{ Pasión } & Deseo & ,856 & 0.933 & $(0.923-0.942)$ \\
\hline & Pasión & ,858 & & \\
\hline & Gusto & ,855 & & \\
\hline & Sexo & ,785 & & \\
\hline & Curiosidad & 627 & & \\
\hline \multirow[t]{8}{*}{ Inseguridad } & Incongruencia & ,626 & 0.861 & $(0.842-0.879)$ \\
\hline & Indiferencia & 609 & & \\
\hline & Ignorancia & ,604 & & \\
\hline & Impotencia &, 505 & & \\
\hline & Baja autoestima &, 574 & & \\
\hline & Cobardía & ,516 & & \\
\hline & Celos & ,467 & & \\
\hline & Egoísmo &, 596 & & \\
\hline \multirow[t]{6}{*}{ Sentimiento de Pérdida } & Enojo &, 582 & .848 & $(0.826-0.868)$ \\
\hline & Tristeza & ,664 & & \\
\hline & Soledad & ,660 & & \\
\hline & Vacío & 678 & & \\
\hline & Miedo & 643 & & \\
\hline & Venganza & ,413 & & \\
\hline \multirow[t]{4}{*}{ Amor hacia otro } & Comprensión por parte de una nueva pareja & ,807 & .881 & $(0.862-0.898)$ \\
\hline & Comunicación con otro(a) & ,812 & & \\
\hline & Cariño hacia una nueva pareja & ,785 & & \\
\hline & Falta de comunicación & ,785 & .786 & $(0.751-0.817)$ \\
\hline \multirow{2}{*}{$\begin{array}{l}\text { Problemas de } \\
\text { Comunicación }\end{array}$} & Desinterés & ,720 & & \\
\hline & Incomunicación & 655 & & \\
\hline
\end{tabular}


Subescala Consecuencias de la Infidelidad

Está compuesta inicialmente por 13 ítems, con un índice de consistencia interna de 0.772 . Contiene dos factores que explican el $46.8 \%$ de la varianza, los cuales se describen a continuación:

1. Consecuencias negativas: se refiere al perjuicio que el acto de infidelidad puede acarrear hacia la relación primaria, propiciando incluso la disolución del vínculo. Su consistencia interna inicial fue 0.909.

2. Consecuencias positivas: se enfoca en el beneficio que el acto de infidelidad puede acarrear hacia la relación primaria, propiciando el acercamiento y la resolución de la problemática dentro del vínculo. Su consistencia interna inicial fue 0.758 .
Al realizar el AFE, se eliminaron los ítems 8 y 10 por comunalidades inferiores a 0.400. En los once ítems restantes, el Alpha de Cronbach fue 0.910; el Determinante 9,23E-005; el KMO, 0,914; y la Prueba de esfericidad de Barlett fue estadísticamente significativa al nivel 0.0001 . En la matriz de correlación Anti-imagen todos los ítems estuvieron por encima de 0.500, mientras que en la varianza total explicada se encontraron dos factores: el primero explica el $54.552 \%$ y el segundo el $21.168 \%$, para un total de $75.720 \%$.

En este análisis los resultados fueron satisfactorios, por lo cual se decide dejar la subescala con 11 ítems. La composición final de esta subescala se muestra en la tabla 4.

Tabla 4. Composición final de la subescala Consecuencias de la Infidelidad

\begin{tabular}{|c|c|c|c|}
\hline Factores & Ítems & Alfa & IC 95\% \\
\hline \multirow[t]{7}{*}{ Consecuencias negativas } & La infidelidad estropea a las parejas & 0.934 & $(0.925-0.942)$ \\
\hline & La infidelidad devalúa a la pareja & & \\
\hline & El tener otra pareja desgasta la relación de pareja & & \\
\hline & La infidelidad destruye las relaciones de pareja & & \\
\hline & La infidelidad siempre es perjudicial para las parejas & & \\
\hline & La infidelidad descubierta provoca celos hacia la pareja & & \\
\hline & La infidelidad descubierta genera desconfianza hacia la pareja & & \\
\hline \multirow[t]{4}{*}{ Consecuencias positivas } & El tener otra pareja ayuda a soportar los problemas del matrimonio & 0.992 & $(0.991-0.993)$ \\
\hline & La infidelidad ayuda a revalorar a la pareja & & \\
\hline & La infidelidad ayuda a mantener a las parejas & & \\
\hline & $\begin{array}{l}\text { Las cadenas de la relación son tan pesadas que a veces se } \\
\text { necesitan } 3 \text { para cargarlas }\end{array}$ & & \\
\hline
\end{tabular}

\section{Discusión}

En el trabajo se presenta un inventario abreviado con la capacidad de medir diferentes categorías de la infidelidad, a través de 4 subescalas: Conducta Infiel, Motivos de Infidelidad, Conceptos de Infidelidad y Consecuencias de la Infidelidad. La primera subescala (conducta infiel) inició con 48 ítems y 4 factores: Infidelidad Sexual, Deseo de Infidelidad Emocional, Deseo de Infidelidad Sexual e Infidelidad Emocional, y posterior al proceso psicométrico, la subescala, finalizó con 23 ítems y los mismos 4 factores. La segunda subescala (Motivos de Infidelidad), inicialmente estuvo compuesta por 70 ítems y 7 factores: Insatisfacción en la Relación Primaria, Sexualidad, Inestabilidad Emocional y Social, Ideología y Normas, Impulsividad, Apatía y Agresión, y después del análisis psicométrico la subescala quedó compuesta por 50 ítems y los mismos factores. La tercera subescala se denomina Concepto de Infidelidad. Inicialmente estaba conformada por 44 ítems y contenía 6 factores: Trasgresión a la Relación, Sentimiento de Pérdida, Insatisfacción, Pasión, Inseguridad, y Amor hacia Otro. Después del proceso psicométrico al que fue sometida la subescala, esta finalizó con 34 ítems y 6 factores, los cuales son Transgresión a la Relación, Pasión, Inseguridad, Sentimiento de Pérdida, Amor hacia Otro, y Problemas de Comunicación. La cuarta y última subescala se denomina (Consecuencias de la Infidelidad). Inicialmente estuvo constituida por 13 ítems y 2 factores, y después de ser sometida al análisis psicométrico quedó conformada por 11 ítems y los mismos factores iniciales.
La infidelidad es un fenómeno multidimensional que genera alteraciones a nivel emocional, psicológico, personal, relacional y social. Respecto a la salud física, Collazos et al. (2005), afirman que la infidelidad es un factor de riesgo para contraer enfermedades de transmisión sexual y $\mathrm{VIH}$, situación que podría convertirse en un factor a tener en cuenta en salud pública.

Al ser un fenómeno relacional, genera múltiples consecuencias en la pareja, siendo la más común el deterioro o ruptura definitiva del vínculo, por lo tanto, las relaciones sexuales extramaritales se constituyen en un gran predictor del divorcio (Amato y Rogers, citado por Nall, Beadle, Newell \& Helm, 2008). En la misma línea, Janus y Janus (1993) hallaron que las relaciones extradiádicas son un factor que provoca el divorcio, en 1 de cada 5 mujeres y en 1 de cada diez hombres que toma esta decisión, posterior a la ocurrencia de dichos problemas.

García, Gómez y Cantó (2001), manifiestan que tanto la infidelidad sexual como la emocional ocasionan reacciones distintas en el hombre y en la mujer. Las reacciones ante la infidelidad sexual en el hombre se caracterizan por una percepción de amenaza a su autoestima, mientras que en la mujer, la infidelidad emocional está determinada por reacciones intensas, con sensación de peligro para la continuidad de la relación. Feeney (2005), quien también estudió las reacciones ante la infidelidad, manifiesta que los sentimientos de dolor evocados por las transgresiones relacionales consecuentes a la devaluación del vínculo, pueden alterar los esquemas de 
valoración positivos en el sí mismo y en el otro.

En ese mismo orden de ideas, Gordon, Snyder y Baucom (2005), manifiestan, que la infidelidad a nivel mental, puede generar sentimientos de vergüenza, depresión, recuerdos dolorosos y evitación, perdiendo predictibilidad sobre el futuro, generando ansiedad y vergüenza. De igual manera, Leary y Springer (2001), expresan que los acontecimientos hirientes producto de esta falta, eventualmente podrían evocar tristeza, generando cólera y amenazas al sentido de seguridad.

Gordon et al. (2005) consideran que cuando la infidelidad no desemboca una ruptura, suelen surgir sentimientos de vergüenza, depresión, recuerdos dolorosos e intrusivos y evitación, planteando que, desde la literatura sobre el trauma, la persona traicionada pierde predictibilidad sobre el futuro, generando ansiedad y vergüenza.

Leary y Springer (2001) exponen que los acontecimientos hirientes que implican la pérdida de relación, eventualmente evocarán la tristeza, cólera y amenazas en el sentido de seguridad, por lo tanto, tal vez produzcan ansiedad. De igual manera Feeney (2005), encuentra que el dolor o la lesión personal son un rasgo que hace parte de la definición de daño.

Otro aspecto a tener en cuenta en los procesos de infidelidad son los celos, los cuales pueden surgir ante una situación real o imaginaria de infidelidad. Harris (2002), demostró que los hombres tienden a sentir más celos asociados al componente sexual que ante el emocional. Sagarin, Becker, Guadagno, Nicastle y Millevoi (2003), dentro de un estudio en el que incluyen variables como la infidelidad con personas del mismo sexo y la experiencia previa de ser víctima de la infidelidad, lograron evidenciar diferencias notables en cuanto al género. Observaron que en los hombres la infidelidad sexual genera mayor incomodidad que la emocional y que el caso contrario ocurre en las mujeres, pero que al evaluar si la infidelidad ocurre con personas del mismo sexo estas diferencias desaparecen.

La versión abreviada del instrumento estudiado (IMIN) posee características satisfactorias de validez y confiabilidad que lo hacen útil en la evaluación de la infidelidad particularmente en el contexto colombiano. Dicha información, permite determinar la conducta infiel representada en el acto infiel sexual o emocional y en el deseo sexual o emocional. Por otro lado, logra indagar sobre las motivaciones para que el individuo ejecute un acto infiel, y explora el concepto que tienen las personas sobre la infidelidad y las consecuencias que este hecho traería para la vida. Cabe resaltar que el uso del inventario esta destinado a la población joven y adulta, etapa evolutiva donde se completa la madurez en concordancia con las transformaciones físicas y psíquicas (Kimmel, Weiner \& Ascaso, 1998; González, Cuellas \& Miguel, 2008).

Será de gran ayuda continuar realizando investigaciones con el instrumento para seguir probando su validez y confiabilidad. En este momento, se considera una buena estrategia de medición que contribuirá a la profundización del fenómeno e incluso su aplicación permitirá tomar decisiones desde la práctica en el manejo de los conflictos relacionales.

\section{Referencias}

Arribas, M. (2004). Diseño y validación de cuestionarios. Matronas profesión, 5(17), 23-29.

Balderrama-Durbin, C., Stanton, K., Snyder, D. K., Cigrang, J. A., Talcott, G. W., Smith Slep, A. M., ... \& Cassidy, D. G. (2017). The risk for marital infidelity across a year-long deployment. Journal of Family Psychology, 31(5), 629.

Banfield, S., \& McCabe, M. P. (2001). Extra relationship involvement among women: Are they different from men?. Archives of Sexual Behavior, 30(2), 119-142.

Barbero García, M. I., Vila Abad, E., \& Holgado Tello, F. P. (2008). La adaptación de los tests en estudios comparativos interculturales. Acción psicológica, 5(2).

Bonilla, M. P. (1993). Infidelidad en la pareja. Conceptualización e implicación en hombres y mujeres mexicanos (Doctoral dissertation, Tesis de Doctorado no publicada. Facultad de Psicología. México: UNAM

Camacho, J. (2004). Fidelidad e infidelidad en las relaciones de pareja. Buenos Aires: Dunken.

Canal, M. \& Carrillo, J. (2013). Análisis De Las Relaciones De Pareja De Los Estudiantes De La Universidad De Pamplona. Cuidado y Ocupación Humana, 1(1).

Carvajal A, Cruz C \& Vásquez M. (1993) Biometría. Cali: Universidad del Valle.

Christensen, A. \& Heavey, C. L. (1999). Intervention for couples. Annual Review of Psychology, 50, 165-90.

Colegio Colombiano de Psicólogos. (2006). Ley 1090: Código Deontológico y Bioético y Otras disposiciones.

Collazos, M. F., Echeverry, N., Molina, A. P., Canaval, G. E., \& Valencia, C. P. (2005). Riesgo de VIH/SIDA en la mujer: no es cuestión de estrato socioeconómico. Colombia Médica, 36(3).

Comrey, A. L., \& Lee, H. B. (2013). A first course in factor analysis. Psychology Press.

Espinoza Romo, A. V., Correa Romero, F. E., \& García y Barragán, L. F. (2014). Percepción social de la infidelidad y estilos de amor en la pareja. Enseñanza e investigación en psicología, 19(1).

Feeney, Judith A (2005) Hurt Feelings In Couple Relationships: Exploring The Role Of Attachment And Perceptions Of Personal Injury. Personal Relationships,12(2), 253-271.

Finchman, F. D. \& Beach, S. R. (1999). Conflict in marriage: Implications for working with couples. Annual Review of Psychology, 30, 47-77.

Frías-Navarro, D., \& Pascual Soler, M. (2012). Prácticas del análisis factorial exploratorio (AFE) en la investigación sobre conducta del consumidor y marketing. Suma Psicológica, 19(1).

García, J. A. (2002). La estructura de la pareja: implicaciones para la terapia cognitivo conductual. Clínica y Salud, 13(1), 89-125.

García, P., Gómez, L. y Cantó, J.M. (2001). Reacción de celos ante una infidelidad: diferencias entre hombres y mujeres y características del rival. Psicothema, 14(1), 611-616.

Glass, S. P., \& Wright, T. L. (1992). Justifications for extramarital relationships: The association between attitudes, behaviors, and gender. Journal of sex Research, 29(3), 361-387.

González, J., Cuéllar, A. \& Miguel, J. (2009). El desarrollo de la identidad en la adolescencia y adultez emergente: 
Una comparación de la identidad global frente a la identidad en dominios específicos. Anales de psicología, 25(2), 316.

Gordon, K. C., Baucom, D. H., \& Snyder, D. K. (2004). An integrative intervention for promoting recovery from extramarital affairs. Journal of Marital and Family Therapy, 30(2), 213-231.

Gordon, K. C., Baucom, D. H. y Snyder, D. K. (2005). Treating couples recovering from infidelity: An integrative approach. Journal of Clinical Psychology, 61: 13931405. doi: 10.1002/jclp.20189

Gottman, J. M. (1998). Psychology and the study of marital processes. Annual Review of Psychology, 49, 169-97

Guitar, A. E., Geher, G., Kruger, D. J., Garcia, J. R., Fisher, M. L., \& Fitzgerald, C. J. (2017). Defining and distinguishing sexual and emotional infidelity. Current Psychology, 36(3), 434-446.

Hair, J. F., Black, W. C., Babin, B. J., Anderson, R. E., \& Tatham, R. L. (1998). Multivariate data analysis . Uppersaddle River. Multivariate Data Analysis (5th ed) Upper Saddle River.

Hair, J. F., Black, W. C., Babin, B. J., Anderson, R. E., \& Tatham, R. L. (2006). Multivariate data analysis 6th ed. Uppersaddle River: Pearson Prentice Hall.

Harris, C. R. (2002). Sexual And Romantic Jealousy In Heterosexual And Homosexual Adults. Psychological Science, 13(1), 7-12.

Janus, S. S., y Janus, C. L. (1993). The Janus report on sexual behavior. New York: Wiley.

Kimmel, D. C., Weiner, I. B., \& Ascaso, L. E. (1998). La adolescencia: una transición del desarrollo. Ariel.

Leary, M. R., y Springer, C. (2001). Hurt feelings: The neglected emotion. In R. M. Kowalski (Ed.), Aversive behaviors and relational transgressions. Washington, DC: American Psychological Association.

Méndez Martínez, C., \& Rondón Sepúlveda, M. A. (2012). Introducción al análisis factorial exploratorio. Revista colombiana de psiquiatría, 41(1).

Merenda, P. F. (1997). Methods, plainly speaking: A guide to the proper use of factor analysis in the conduct and reporting of research: Pitfalls to avoid. Measurement and Evaluation in counseling and Development, 30(3), 156.

Montero, I., \& León, O. G. (2007). Guía para nombrar los estudios de investigación en Psicología. International Journal of Clinical and Health Psychology, 7(3), 847862.

Nall, B., Beadle, M., Newell, J. y Helm, H. M. (2008). Spousal Disclosure of Extramarital Relationships: Attitudes of Marriage and Family Therapists. The Family Journal: Counseling And Therapy For Couples And Families, 16(4), 328-337.

Oviedo, H. C., \& Arias, A. C. (2005). Aproximación al uso del coeficiente alfa de Cronbach. Revista colombiana de psiquiatría, 34(4), 572-580.

Pérez, E. R., \& Medrano, L. (2010). Análisis factorial exploratorio: bases conceptuales y metodológicas. Revista Argentina de Ciencias del Comportamiento, 2(1). 58-66

Pittman, F. S. (2003). Mentiras privadas: la infidelidad y la traición de la intimidad. 1a reimp. Buenos Aires, Amorrortu.

Romero Palencia, A., Rivera Aragón, S., \& Díaz Loving, R. (2007). Desarrollo del inventario multidimensional de infidelidad (IMIN). Revista Iberoamericana de Diagnóstico y Evaluación-e Avaliação Psicológica, 1(23).

Sagarin, B. Becker, D,V.Guadagno, R. Nicastle, L. Millevoi, A.(2003). Sex differences (and similarities) in jealousy: The moderating influence of infidelity experience and sexual orientation of the infidelity. Evolution y Human Behavior, 24(1), 17-23.

Scheinkman, M., \& Fishbane, M. (2004). The vulnerability cycle: Working with impasses in couples therapy. Family Process, 43, 279-299. En http://www.ncbi. nlm.nih.- gov/pubmed/15386955

Sepúlveda, F. M. (2010). Validez de los Tests y el Análisis Factorial: Nociones Generales. Ciencia \& Trabajo, 12(35).

Weis, D. L., \& Felton, J. R. (1987). Marital exclusivity and the potential for future marital conflict. Social Work, 32(1), 45-49.

Weiser, D. A., Lalasz, C. B., Weigel, D. J., \& Evans, W. P. (2014). A prototype analysis of infidelity. Personal Relationships, 21(4), 655-675.

Whisman, M. A. (1999). Marital dissatisfaction and psychiatric disorders: Results from the National Comorbidity Survey. Journal of Abnormal Psychology, 108, 701706

Whisman, M. A., \& Wagers, T. P. (2005). Assessing relationship betrayals. Journal of clinical psychology, 61(11), 1383-1391.

Yeniceri, Z., \& Kökdemir, D. (2006). University Students'perceptions Of, And Explanations For, Infidelity: The Development Of The Infidelity Questionnaire (Infq). Social Behavior \& Personality: an international journal, 34(6).

Zola, M. F. (2007). Beyond infidelity-related impasse: An integrated, systemic approach to couples therapy. Journal of systemic therapies, 26(2), 25-41.

Zumaya, M., Brown, C., \& Baker, H. (2008). Las parejas y sus infidelidades. Med Sur, 15(3), 225-230 